\title{
Data Security in Cloud Environments
}

\author{
V. Bhavana1)
}

\begin{abstract}
Intentional or unintentional leakage of confidential data is without a doubt a standout amongst the most serious security dangers that associations confront in the computerized period. The danger now reaches out to our own lives: a plenty of individual data is accessible to informal communities and advanced mobile phone suppliers and is in a roundabout way exchanged to dishonest outsider and fourth gathering applications. In this work, we display a non specific information heredity structure LIME for information stream over various substances that take two trademark, chief parts (i.e., proprietor and shopper). We characterize the correct security ensures required by such an information ancestry system toward ID of a blameworthy element, and recognize the improving non-revocation and trustworthiness presumptions. We then create and break down a novel responsible information exchange convention between two substances inside a noxious situation by expanding upon unaware exchange, powerful watermarking, and mark primitives. At long last, we play out a test assessment to exhibit the reasonableness of our convention and apply our system to the imperative information spillage situations of information outsourcing and interpersonal organizations. All in all, we consider LIME, our genealogy system for information exchange, to be a key stride towards accomplishing responsibility by plan.
\end{abstract}

Keywords: information leakage, data lineage, accountability, algorithm, fingerprinting, watermarking.

\section{Introduction}

In the computerized period, data spillage through accidental exposures, or deliberate harm by displeased workers and malignant outside elements, display a standout amongst the most genuine dangers to associations. As indicated by a fascinating sequence of information breaks kept up by the Privacy Rights Clearinghouse (PRC), in the United States alone, 868;045;823 records have been broken from 4;355 information ruptures made open since 2005 [1-10]. It is not hard to trust this is recently the tip of the icy mass, as most instances of data spillage go unreported because of dread of loss of client certainty or administrative punishments: it costs organizations by and large \$214 per bargained record [11-20]. A lot of computerized information can be replicated at no cost and can be spread through the web in brief time. Furthermore, the danger of getting got for information spillage is low, as there are presently

Received(July 20, 2015), Review Result(1st: August 7, 2015, 2nd: September 14, 2015), Accepted(December 10, 2015)

${ }^{1}$ (Corresponding Author) Machine Intelligence Research Labs, India

email: bhavanatwinky@gmail.com 
no responsibility systems. Hence, the issue of information spillage has achieved another measurement these days. Not just organizations are influenced by information spillage, it is likewise a worry to people. The ascent of interpersonal organizations and cell phones has exacerbated things. In these situations, people unveil their own data to different specialist co-ops, regularly known as outsider applications, as an end-result of some perhaps free administrations. Without appropriate directions and responsibility instruments, a large portion of these applications impart people's recognizing data to many promoting and Internet following organizations. Indeed, even with get to control systems, where access to touchy information is restricted, a malignant approved client can distribute delicate information when he gets it. Primitives like encryption offer security just the length of the data of intrigue is encoded, yet once the beneficiary decodes a message, nothing can keep him from distributing the unscrambled content. Along these lines it appears to be difficult to anticipate information spillage proactively.

\section{Proposed system}

\subsection{Related work}

A preparatory shorter rendition of this paper showed up at the STM workshop [21]. This adaptation constitutes a noteworthy augmentation by including the accompanying commitments: We give a more point by point depiction of our model, a formal determination of the utilized primitives, an investigation of the presented convention, a talk of execution results, a use of our structure to case situations, a dialog of extra components and an amplified exchange of related work.

\subsection{Other Models}

Hasan et al. [22] exhibit a framework that authorizes logging of read and compose activities in a sealed provenance chain. This makes the likelihood of checking the cause of data in an archive. Notwithstanding, as an assailant can piece of the provenance data of a document, the issue of information spillage in pernicious situations is not handled by their approach. The model acquainted in [8] plans with assist the information wholesaler with identifying the pernicious specialist which released the data. Moreover, they contend that ebb and flow watermarking procedures are not functional, as they may implant additional data which could 
influence operators' work and their level of strength might be lacking. In LIME the relationship of information wholesaler and operators compares to the relationship between information proprietor and shopper and the model could be utilized as an option technique to follow the data given to the customers.

\subsection{Other Fingerprinting Protocols}

In Poh addresses the issue of responsible information exchange with untrusted senders utilizing the term reasonable substance following. He displays a general system to look at changed methodologies and parts conventions into four classes relying upon their use of trusted outsiders, i.e., no trusted outsiders, disconnected trusted outsiders, online confided in outsiders and put stock in equipment. Besides, he presents the extra properties of beneficiary obscurity and decency in relationship with installment. All displayed plans utilize watermarking to follow the liable party and most introduced conventions make utilization of watermarking in the scrambled space, where encoded watermarks are inserted in scrambled records. A noteworthy preferred standpoint of our plan is that it can be utilized with each current watermarking plan with no change. The plans depending on watermarking in the scrambled area just work with watermarking plans that are intended for this strategy. Another plan displayed depends on chameleon encryption. In Sadeghi additionally looks at a few fingerprinting plans and introduces new developments for symmetric, unbalanced and mysterious fingerprinting plans. The topsy-turvy conspire utilizes a homomorphic responsibility plan to figure the fingerprinted variant of the report.

\subsection{Existing system}

In the digital era, data spillage through unintentional exposures, or intentional sabotage by disappointed representatives and malevolent outside substances, show a standout amongst the most genuine dangers to associations. It is not hard to trust this is recently the tip of the ice sheet, as most instances of data spillage go unreported because of dread of loss of client certainty or administrative punishments Large measures of digital information can be replicated at no cost and can be spread through the web in brief time. Furthermore, the danger of getting got for information spillage is low, as there are at present no responsibility components. Therefore, the issue of information spillage has achieved a new dimension nowadays [23-25]. 


\subsection{Disadvantages}

Indeed, even with access control mechanisms, where access to delicate information is restricted, a malevolent approved client can distribute touchy information when he gets it. Primitives like encryption offer security just the length of the data of intrigue is encoded, yet once the beneficiary unscrambles a message, nothing can keep him from distributing the decoded content. Along these lines it appears to be difficult to prevent information spillage proactively.

\subsection{Proposed System}

Propose different arrangements; the previous gives the sender a chance to open a few sets to approve that they are not equivalent and the last uses unaware exchange with a two-bolt cryptosystem where the beneficiary can think about both forms in encoded shape. Be that as it may, both proposed arrangements have a few defects themselves. The issue is that it is conceivable to make two unique renditions with a similar watermark, so regardless of the possibility that the uniformity test falls flat, the two offered variants can even now have a similar watermark and the sender will know which watermark the beneficiary got. Additionally, the settle proposed in remains the unimportant likelihood of disappointment, as it doesn't part the archive into parts, yet makes $\mathrm{n}$ distinctive. We see that all topsy-turvy fingerprinting conventions in view of careless exchange that have been proposed so far experience the ill effects of a similar shortcoming. We go around this issue in our convention by also sending a marked message including the watermark's substance, so that the beneficiary can demonstrate what he requested. In contrast to the watermark, this message can be read by the recipient, so he can notice if the sender cheats.

\subsection{Advantage}

The former gives the sender a chance to open a few sets to approve that they are not equivalent and the last uses careless exchange with a two-bolt cryptosystem where the beneficiary can think about both forms in encoded shape. Be that as it may, both proposed arrangements have a few blemishes themselves. The issue is that it is conceivable to make two distinct adaptations with a similar watermark, so regardless of the possibility that the equity 
test comes up short, the two offered renditions can at present have a similar watermark and the sender will know which watermark the beneficiary received.

\section{Implementation Modules}

\subsection{Micro benchmarking}

We enforced the protocol in as a proof-of-concept and to research its performance. For the oblivious transfer sub protocol we tend to enforced the protocol] exploitation the PBC library, that itself makes use of the GMP library. For signatures we tend to enforced the BLS theme, additionally exploitation the PBC library. For centrosymmetric encoding we tend to used associate degree implementation of AES from the Crypto++ library. For watermarking we tend to used associate degree implementation of the Cox algorithmic program for strong image we tend to set the -factor, that determines the strength of the watermark, to a price of zero.1. we tend to dead the experiment with totally different parameters to research the performance. The sender and recipient a part of the protocol area unit each dead within the same program, i.e., we tend to don't analyze network causing, however solely procedure performance. The death penalty machine may be a Lenovo ThinkPad model T430 with eight GB RAM and four $\times$ a pair of.6GHz cores, however all executions were performed consecutive. we tend to measured execution times for various phases of the protocol: watermarking, signature creation, encryption, oblivious transfer and detection. we tend to dead every experiment 250 times and determined the common computation time and also the variance.

\subsection{Possible knowledge Distortion}

we used an easy rending algorithm: we tend to split the image into $n$ equally sized squares. However, after we used a powerful watermark for the tiny elements (that is that the - issue utilized by the Cox algorithmic program is zero.5), variations between adjacent elements became visible even if the one watermarks area unit impalpable. The ensuing image. This impact becomes even stronger when multiple iterations as determined. In some cases, this distortion would possibly have an effect on the usability of the document. we tend to stress but, that we tend to were still able to get smart results with our approach. In Fig. 5(a1) we tend to used 
the Cox algorithmic program with associate degree alpha issue of zero.1 and no distortion is visible. it'd be attention-grabbing to research if this drawback are often circumvented by exploitation additional elaborate rending algorithms. As most watermarking schemes create use of the adjacency of data within the document, this is often not a trivial task.

\section{Conclusion}

We introduce LIME, a model for responsible information exchange over various elements. We characterize taking an interest gatherings, their between connections and give a solid instantiation for an information exchange convention utilizing a novel mix of neglectful exchange, vigorous watermarking and advanced marks. We demonstrate its accuracy and demonstrate that it is feasible by giving microbenchmarking comes about. By exhibiting a general relevant structure, we present responsibility as right on time as in the outline period of an information exchange foundation.

In spite of the fact that LIME does not effectively anticipate information spillage, it presents responsive responsibility. Along these lines, it will prevent pernicious gatherings from releasing private records and will energize fair (yet reckless) gatherings to give the obliged insurance to delicate information. LIME is adaptable as we separate between put stock in senders (generally proprietors) and untrusted senders (normally buyers). On account of the confided in sender, an exceptionally basic convention with minimal overhead is conceivable. The untrusted sender requires a more entangled convention, yet the outcomes are not in view of trust presumptions and in this way they ought to have the capacity to persuade an unbiased substance (e.g., a judge).

Our work additionally inspires additionally explore on information spillage identification procedures for different report sorts and situations. For instance, it will be a fascinating future research course to plan an obvious heredity convention for inferred information. 


\section{References}

[1] http://www.privacyrights.org/data-breach/, Chronology of data breaches, May 5 (2014).

[2] http://www.symantec.com/about/news/release/article.jsp?prid=20110308_01/, Data breach cost, July 2 (2011).

[3] http://www.privacyrights.org/, Privacy rights clearinghouse, Jun 20 (2014).

[4] http://epic.org/, Electronic privacy information center (EPIC), January 13 (1994).

[5] http://online.wsj.com/article/SB10001424052702304772804575558484075236968.html,/ Facebook in privacy breach, May 5 (2010).

[6] http://www.computerworld.com/s/article/109938/Offshore_outsourcing_cited_in_Florida_data_leak/, January 13 (1994).

[7] A. Mascher-Kampfer, H. Stögner, and A. Uhl, Multiple Re-Watermarking Scenarios, Proc. 13th Int. Conf. Syst., Signals, Image Process., (2006), pp.53-56.

[8] P. Papadimitriou and H. Garcia-Molina, Data leakage detection, IEEE Trans. Knowl. Data Eng., (2011), Vol.23, No.1, pp.51-63.

[9] http://crypto.stanford.edu/pbc/, Pairing-based cryptography library (PBC) Jun 20 (2014).

[10] I. J. Cox, J. Kilian, F. T. Leighton, and T. Shamoon, Secure spread spectrum watermarking for multimedia, IEEE Trans. Image Process., (1997), Vol.6, No.12, pp.1673-1687.

[11] B. Pfitzmann and M. Waidner, Asymmetric fingerprinting for larger collusions, in Proc. 4th ACM Conf. Comput. Commun. Security, (1997), pp.151-160.

[12] S. Goldwasser, S. Micali, and R. L. Rivest, A digital signature scheme secure against adaptive chosen-message attacks, SIAM J. Comput., (1988), Vol.17, No.2, pp.281-308.

[13] A. Adelsbach, S. Katzenbeisser, and A.-R. Sadeghi, A computational model for watermark robustness, in Proc. 8th Int. Conf. Inf. Hiding, (2007), pp.145-160.

[14] J. Kilian, F. T. Leighton, L. R. Matheson, T. G. Shamoon, R. E. Tarjan, and F. Zane, Resistance of digital watermarks to collusive attacks, in Proc. IEEE Int. Symp. Inf. Theory, (1998), pp.271-271.

[15] M. Naor and B. Pinkas, Efficient oblivious transfer protocols, in Proc. 12th Annu. ACM-SIAM Symp. Discrete Algorithms, (2001), pp.448-457.

[16] http://gmplib.org/, GNU multiple precision arithmetic library (GMP), (2014).

[17] D. Boneh, B. Lynn, and H. Shacham, Short signatures from the Weil pairing, in Proc. 7th Int. Conf. Theory Appl. Cryptol. Inf. Security: Adv. Cryptol., (2001), pp.514-532.

[18] http://cryptopp.com/, W. Dai. Crypto++ Library, January 13 (2013).

[19] http://www.cosy.sbg.ac.at/ pmeerw/Watermarking/source/, P. Meerwald. Watermarking toolbox, July 2 (2010).

[20] Y. Ishai, J. Kilian, K. Nissim, and E. Petrank, Extending oblivious transfers efficiently, in Proc. 23rd 
Annu. Int. Cryptol. Conf. Adv. Cryptol., (2003), pp.145-161.

[21] M. Backes, N. Grimm, and A. Kate, Lime: Data lineage in the malicious environment, in Proc. 10th Int. Workshop Security Trust Manage., (2014), pp.183-187.

[22] R. Hasan, R. Sion, and M. Winslett, The case of the fake picasso: Preventing history forgery with secure provenance, in Proc. 7thConf.FileStorageTechnol., (2009), pp.1-14.

[23] A. Pretschner, M. Hilty, F. Schütz, C. Schaefer, and T. Walter, Usage control enforcement: Present and future, IEEE Security Privacy, (2008), Vol.6, No.4, pp.44-53.

[24] F. Kelbert and A. Pretschner, Data usage control enforcement in distributed systems, Proc. 3rd ACM Conf. Data Appl. Security Privacy, (2013), pp.71-82.

[25] F. Salim, N. P. Sheppard, and R. Safavi-Naini, A rights management approach to securing data distribution in coalitions, Proc. 4th Int. Conf. Netw. Syst. Security, (2010), pp.560-567. 